NASA Contractor Report 4288

\title{
Performance of an Optimized Rotor Blade at Off-Design Flight Conditions
}

Aditi Chattopadhyay

Analytical Services \& Materials, Inc. Hampton, Virginia

Prepared for

Langley Research Center under Contract NAS1-18599

\section{N/SN}

National Aeronautics and

Space Administration

Office of Management

Scientific and Technical

Information Division

1990 



\title{
Performance of an Optimized Rotor Blade at Off-Design Flight Conditions
}

\author{
by \\ Aditi Chattopadhyay \\ Analytical Services \& Materials, Inc. \\ Hampton, Virginia 23666
}

\begin{abstract}
An investigation is made of the dynamic and aerodynamic performance of a helicopter rotor previously optimized for minimum 4/rev vertical shear and blade weight subject to certain dynamic and structural constraints. The program CAMRAD which was used in designing the optimized blade is used for both dynamic and aerodynamic analysis. The behavior of the optimized rotor is analyzed over a wide range of operating conditions and for a larger number of rotor characteristics than those considered in designing the blade. To assess the dynamic behavior, the blade root vibratory shears and moments that are transmitted to the rotor hub are calculated. The aerodynamic performance assessments are made based on the power required by the rotor for a given rotor task, the rotor lifting efficiencies, maximum rotor thrust envelopes and the control margins. Results are presented for the optimized blade and the reference blade, which was used as the baseline for the optimized blade, for two rotor tasks.
\end{abstract}

\section{Nomenclature}

$\begin{array}{ll}\mathrm{c}_{1} & \text { blade segment lift coefficient } \\ \mathrm{c}_{\mathrm{r}} & \text { root chord, } \mathrm{ft} \\ \mathrm{f}_{3}, \mathrm{f}_{4}, \mathrm{f}_{5}, \mathrm{f}_{6} & \text { natural frequencies of first four coupled elastic modes } \\ \mathrm{k}_{\mathrm{r}} & \text { principle radius of gyration at blade root, } \mathrm{ft} \\ \mathrm{x}, \mathrm{y}, \mathrm{z} & \text { reference axes }\end{array}$

1. Research Scientist, Member AIAA, AHS. 


\begin{tabular}{|c|c|}
\hline$C_{L}$ & lift coefficient \\
\hline$c_{x}$ & propulsive force coefficient \\
\hline$C_{P}$ & total power coefficient \\
\hline & induced power coefficient \\
\hline & profile power coefficient \\
\hline $\mathrm{Q}$ & torque coefficient \\
\hline $\mathrm{C}_{\mathrm{T}}$ & thrust coefficient \\
\hline $\mathrm{D}$ & drag, lb \\
\hline $\mathrm{EI}_{\mathrm{xx}}, \mathrm{EI}_{\mathrm{zz}}$ & bending stiffnesses at blade root, $l \mathrm{~b}-\mathrm{ft}^{2}$ \\
\hline $\mathrm{F}_{\mathrm{z}}$ & thrust component in $\mathrm{z}$ direction, $\mathrm{lb}$ \\
\hline $\mathrm{GJ}_{\mathrm{r}}$ & torsional stiffness at blade root, $1 \mathrm{~b}-\mathrm{ft}^{2}$ \\
\hline $\mathrm{L}$ & lift, lb \\
\hline Q & rotor torque, lb-ft \\
\hline $\mathrm{R}$ & blade radius, $\mathrm{ft}$ \\
\hline $\mathrm{T}$ & rotor thrust, lb \\
\hline $\mathrm{x}$ & rotor propulsive force, $\mathrm{lb}$ \\
\hline$\lambda$ & taper ratio \\
\hline$\mu$ & advance ratio \\
\hline$\sigma$ & area solidity \\
\hline & rotor angular velocity, $\mathrm{rad} / \mathrm{sec}$. \\
\hline
\end{tabular}

\section{Introduction and Background}

Currently at NASA Langley Research Center, there is an effort to integrate various disciplines in the rotor blade design process ${ }^{1}$. A first investigation at integrating some of these disciplines is reported in Ref. 2, where the integration of aerodynamic loads effects and the dynamic aspects of blade design was addressed by coupling a comprehensive helicopter analysis code, CAMRAD ${ }^{3}$, to an optimizer consisting of the nonlinear optimization algorithm, $\mathrm{CONMIN}^{4}$, and an approximate analysis technique. A combination of the blade root $4 / \mathrm{rev}$ vertical shear and the blade weight was minimized, for a four-bladed rotor, using a multiple objective function technique. Constraints were imposed on the blade natural frequencies, the autorotational inertia of the blade and the centrifugal stress. The use of the program CAMRAD permitted the design of the blade under calculated airloads and its presence in the closed loop 
optimization procedure allowed the inclusion of changes in these airloads with changes in design variables during optimization. The paper demonstrated a significant reduction in the $4 / \mathrm{rev}$ vertical shear and blade weight along with overall reductions in the amplitudes of the oscillatory vertical airloads, azimuthally and radially, for the optimized blade when compared to a baseline ('reference') blade. As a byproduct, it was shown that optimization also reduced the total power required by the rotor while maintaining the same $\mathrm{C}_{\mathrm{T}} / \sigma, \mathrm{C}_{\mathrm{T}}$ being the rotor thrust coefficient and $\sigma$ the thrust weighted solidity of the rotor. The optimization in Ref. 2 was performed for the rotor in forward flight at an advance ratio $\mu=0.3$ subject to a reasonable but limited set of design constraints. For example only $4 / \mathrm{rev}$ vertical shear was involved and no constraint was imposed on the total rotor thrust, although the $C_{T} / \sigma$ was held constant between the reference and the optimized blades. This led to an overall reduction in blade thrust after optimization due to reduction in the solidity of the optimized rotor.

It was of interest to determine how well a blade designed for one flight condition and a limited number of constraints would perform for other conditions and to assess performance with respect to criteria not included in the design process. In this paper the optimized blade design from Ref. 2 is investigated in detail. The performance of the optimized blade designed at a prescribed flight condition is studied at other flight conditions. The behavior of the optimized blade is studied with respect to the dynamic and aerodynamic performance criteria not included in the optimization formulation. These studies are conducted by taking the optimized blade design and analyzing its off-design characteristics by performing a detailed aerodynamic and dynamic analyses using CAMRAD.

\section{The Optimized Blade}

A brief description of the optimized blade along with the basis for obtaining it is presented in this section. The optimization procedure developed in Ref.2 was applied to a baseline ('reference') blade. The reference blade was a modified version of a wind tunnel model of an

advanced rotor blade ${ }^{5}$. The reference and the optimized blades (Fig. 1) are articulated with rigid hubs and have linear twist distributions. The blade was optimized for minimum weight and minimum 4/rev vertical root shear. The following constraints were imposed:

upper and lower bounds on first four elastic coupled blade natural frequencies

lower bound on blade autorotational inertia

upper bound on centrifugal stress on each blade segment

The design variables were the blade bending and torsional stiffnesses, nonstructural masses, chord, radius of gyration and taper ratio. Blade radius, $R$, rotor angular velocity, $\Omega$, airfoil distributions and hinge off-sets were fixed during optimization. These preassigned parameters are listed in Table 1. A comparison of the optimized and the reference blade is presented in Table 2 where it can be seen that the reference blade has a rectangular planform (Fig. 1) whereas the optimized blade is tapered with a taper ratio $\lambda=1.33$. 


\section{Study Description}

The intent of the present paper is to investigate the loads and performance behavior of the optimized rotor over a wide range of operating conditions and for a large number of rotor characteristics than those considered in the design process, in order to assess the design. This is being accomplished by conducting a two-part study of the 'off-design' behavior of the optimized rotor as it compares with the reference rotor. The two parts of the study address blade loads (Part 1) and rotor aerodynamic performance (Part 2). Rotor aerodynamic performance issues are addressed here even though they were not considered in the original optimization.

The program CAMRAD is used for calculating the vibratory airloads for the optimized and the reference blades. In CAMRAD, the blade response is computed using rotating, free-vibration

modes, equivalent to a Galerkin analysis ${ }^{6}$. Ten bending modes, of which seven are flapping (one rigid and six elastic), three are lead-lag (one rigid and two elastic) and one is a rigid body torsion mode, are calculated. Main blade resonances up to eight per revolution are included and therefore, eight harmonics of the rotor revolution are retained in the air loads calculation. The blade loads are generated using the isolated rotor model in CAMRAD.

CAMRAD is also used to predict the performance of the optimized and the reference rotors. The code is run using the same aerodynamic assumptions used in the optimization study. These assumptions include: uniform inflow, yawed flow on the rotor, unsteady aerodynamics and no dynamic stall. It is recognized that some of these assumptions will produce an inaccurate picture of the rotor aerodynamic environment (particularly the inflow assumption), however, the authors feel that it is more appropriate to retain the same model for the purpose of this theoretical study.

\section{Part 1: Blade Loads Study}

For a four-bladed rotor, the critical vibratory blade loads, that are transmitted to the rotor hub and therefore need to be reduced, are listed below:
1) $4 / \mathrm{rev}$ vertical shear,
2) 3 and $5 / \mathrm{rev}$ inplane shear,
3) 3 and $5 /$ rev radial shear,
4) 3 and 5/rev flapping moment,
5) 3 and $5 / \mathrm{rev}$ torsional moment,
6) $4 /$ rev lagging moment.

The $4 / \mathrm{rev}$ vertical shear produce oscillatory $4 / \mathrm{rev}$ loads in the vertical direction at the hub. In the optimized blade, the minimization of this shear force was done at a particular flight condition, therefore, its value at 'off-design' flight conditions needs to be calculated. The purpose of this study is to see whether the value of the shear force for the optimized blade remains lower than the reference blade value at other flight conditions. The 3 and $5 / \mathrm{rev}$ components of the inplane and the radial shears at the blade root are transmitted as $4 / \mathrm{rev}$ forces in both lateral and longitudinal directions at the rotor hub. Since they were not used as objective functions or as constraints, it is of interest to compute their values for both the optimized and the 
reference blades. The 3 and 5/rev components of the flapping and the torsional moments at blade root affect the rotor hub in the form of oscillatory $4 / \mathrm{rev}$ pitch and roll moments. These were not included in the optimum design process of Ref. 2 but are nevertheless critical from an airframe vibration point of view. Finally, the $4 / \mathrm{rev}$ blade root lagging moment, which causes oscillatory $4 / \mathrm{rev}$ yaw moment at the rotor hub is also an essential ingredient in rotor blade vibration evaluation and therefore is computed for both the rotors.

\section{Part 2: Performance Study}

In this study, performance of the optimized blade is based on four criteria:

1) rotor power required for a given task,

2) rotor lifting efficiency $L / D$,

3) load factor envelope,

4) control margins.

Rotor power required for a given task is primarily a measure of economic efficiency. Power requirements for a given rotor task size the engine and drive the fuel flow both of which affect weight and operating cost. The CAMRAD computer code predicts the rotor torque based on the summation of forces on the rotor hub. It is useful to split the power required according to the type of energy loss. This allows the comparison of components of the rotor power and reveals how the performance gains are achieved. For the present study, induced power, $\mathrm{C}_{\mathrm{P}_{\mathrm{i}}}$, and profile power, $\mathrm{C}_{\mathrm{P}_{\mathrm{p}}}$, are important. These quantities define the power required to lift and rotate the rotor through the air under the specified conditions.

Rotor lifting efficiency is measured by $L / D$. This quantity is also obtained from the CAMRAD code. The load factor envelope is the limit with respect to speed of maximum attainable thrust for a given propulsive force, divided by the design thrust. This gives a measure of the steady turning capability or the maximum sustained thrust of the rotor. These values are obtained by increasing the lift requirement for the rotor until the power required diverges or the rotor stalls (this usually happens at about the same time).

Rotor control margin is a measure of the control inputs required to achieve a particular trimmed solution. In general, it is good to minimize the amount of control displacement in order to provide an extra 'margin'. For example, an aircraft trimmed at high speed which uses all of its control displacement to achieve this state is not capable of any type of maneuver around this condition and can only retreat to a less demanding condition. An improved design could alleviate this problem by reducing the required control input.

These criteria are used to judge the relative merits of the optimized rotor. In addition, the comparisons are made over a wide range of operating conditions including speed and thrust variations covering the entire operating envelope of the rotor. It should be noted that this rotor was not optimized for any performance condition and any improvement should be viewed as a synergistic result of the optimization. 


\section{$\underline{\text { Results }}$}

The studies on the airloads and rotor performance are conducted for both the reference and the optimized blade. CAMRAD is used to obtain a power profile of the reference blade as shown in Fig. 2. The power profile is necessary for determining the feasible range of advance ratio values for the rotor in which its dynamic and aerodynamic performance is to be studied. The results obtained are categorized into two sections, loads and performance.

\section{Loads Results}

For a meaningful study of the two rotors, the loads comparison will be made for two rotor tasks. Task 1 requires the optimized rotor to produce the same $\mathrm{C}_{\mathrm{T}} / \sigma$ and $\mathrm{C}_{\mathrm{X}} / \sigma$ as the reference rotor. Task 2 requires the optimized rotor to produce the same thrust, $T$, and the same propulsive force, $\mathrm{X}$, as the reference rotor. Using these two tasks provides a comparison on both a nondimensional basis, for which the rotor was optimized, and a dimensional basis, for which the rotor might be expected to operate, and compares the rotors' ability to produce lift with low induced power and to produce high lift without stalling.

The optimized rotor was designed to provide minimum $4 / \mathrm{rev}$ vertical shear at blade root and minimum blade weight under forward flight condition at an advance ratio of $\mu=0.30$. A study was done to estimate the $4 / \mathrm{rev}$ vertical shear over a wide range of advance ratio values $(\mu=$ $0.05-0.40$ ). Figure 3 presents the $4 / \mathrm{rev}$ vertical root shear for the reference blade and the optimized blade, operating at both rotor tasks. The figure indicates that the optimized blade has lower vertical shear than the reference blade and the reduction is more significant at higher speeds. The maximum reduction occurs at $\mu=0.3$, the design point. The reduction in the vertical shear is partly due to the reduced thrust in Task 1 where the $\mathrm{C}_{\mathrm{T}} / \sigma$ is fixed and the rotor thrust reduces due to reduction in solidity after optimization. However, the optimized rotor operating at Task 2 also has significant reductions in the vertical shear, over the reference rotor, for most of the speed range. Of particular interest is the smaller vertical shear in the optimized blade for Task 2 than for Task 1 . This is because the two rotors (reference and optimized) are operating at the same $T$ in Task 2 and the optimized rotor having a much smaller $\sigma$, is operating at a very high value of $C_{T} / \sigma$. Thus at high speeds, due to higher thrust requirements that can drive the rotor to stall, there is a large increase in the vertical shear.

Certain shear forces which were not included in the optimized blade design, are calculated for various values of $\mu$. Figures 4 and 5 present the $3 / \mathrm{rev}$ components (the $5 / \mathrm{revs}$ components were much smaller and are not shown) of the inplane and the radial shears at the blade root. The figures indicate that the optimized blade designed for minimum 4/rev vertical shear also has lower values of the critical inplane and radial shears at both rotor tasks. The inplane and the radial shears also have very similar variations with speed. These figures also show that the differences between the reference and the optimized blade shear force values amplify with speed.

The critical root bending and torsional moments are also calculated for the same speed range. Figures 6 and 7 respectively present the $3 / \mathrm{rev}$ flapping and the torsional moments at the 
blade root for the reference and the optimized blade. The figures indicate reduction in these moments for the optimized blade over the entire speed range at both rotor tasks. The lowest values of course occur at Task 1 when the rotor is operating under reduced thrust. It is interesting to note the nature of the flapping moment from Fig. 6 which shows occasional reductions at certain speeds, e.g., at $\mu=0.25$ the value is lower than that at $\mu=0.30$ for the optimized rotor under Task 1 . This is due to modal cancellation, i.e., the forcing function becoming orthogonal to one of the mode shapes. Figure 8 presents the 4/rev lagging moment variation with speed. For the optimized rotor for Task 1 the lagging moment is lower than the optimized blade as expected, with the differences magnified at higher speeds. However, for Task 2 there is a crossover at a higher speed. In other words, the lagging moment for the optimized blade becomes higher than the reference blade at high speeds. This is because the $4 / \mathrm{rev}$ lagging moment is responsible for the 4/rev yawing moment at the rotor hub which increases with increase in thrust (recalling that higher speed requires higher thrust). Once again, at certain speeds, the figure shows occasional reductions of the lagging moment for both the reference and the optimized blades possibly occurring due to modal cancellation.

In summary, the optimized blade designed to reduce $4 / \mathrm{rev}$ vertical shear and blade weight also reduces most other critical root shears and bending moments for the speed range studied. Some of the reductions are due to the lower thrust requirements in the optimized rotor which was designed to satisfy Task 1 . However, the optimized rotor for Task 2 retained most of the reductions in the vibratory loads at lower speeds.

\section{Performance Results}

A comparison between the aerodynamic performance of the two rotors is made for the same two tasks as the loads study. Recall that Task 1 compares the two rotors at the same $\mathrm{C}_{\mathrm{T}} / \sigma$, hence this task is useful in comparing the lifting efficiency of the two rotors. Task 2 , on the other hand, compares the rotors at the same $\mathrm{T}$, which is meaningful in comparing lifting capability.

Figures $9-11$ present the comparison of results for the two rotors for Task 1 . The comparison is made for advance ratios ranging from 0.05 to 0.40 . Figure 9 presents the total power required (normalized with respect to $\sigma$ ) versus advance ratio. Figures 10 and 11 present the breakdown of the predicted power into induced and profile components. These figures clearly show an improvement in total power required for the conditions studied. Of particular interest is the marked reduction in total power required at the lower advance ratios (e.g. far from the optimization point). Figure 10 presents the normalized induced power comparisons which shows the source of this reduction. Recall that for Task 1 the optimized rotor is in fact operating at a lower value of $C_{T}$ and hence requires less induced power. This difference would be expected to be more pronounced at lower speeds where the induced power requirements are generally higher. Figure 11 presents the normalized profile power comparison between the two rotors. For Task 1, both rotors are operating at the same mean $C_{L}$, therefore the normalized profile drag on the rotors should be very similar since both rotors have the same aiffoil section. However, since the optimized rotor is tapered it has an advantage. The advantage lies in the fact that the profile 
drag losses are proportional to the cube of the velocity, whereas, the thrust varies as the square of the velocity, making it advantageous for the thrust to be produced by the low velocity inboard sections. For two rotors operating at the same $C_{T} / \sigma$, the advantage would lie with the rotor which produces its lift from more inboard sections. This is precisely the effect that taper produces. Figure 11 shows that the optimized rotor does have the advantage in profile power.

Figures 12 - 14 present the comparison of the two rotors for Task 2. Here again, the optimized blade requires less power, however the differences between the two are not as large. In Fig. 12 the two power curves are much closer together at both the high speed and low speed parts of the power polar (e.g. far from the optimum design point) and only in the middle part of the range (e.g. near the optimum design point) is there any great difference. Recall that for Task 2 , both rotors are operating at the same $T$, hence the optimized rotor has no advantage in induced power requirements. Figure 13 presents the comparison of induced power requirements and indeed shows that the optimized rotor requires slightly more induced power that the reference rotor. By contrast, Fig. 14 presents the profile power comparison of the two rotors and indicates a significant decrease for the optimized blade. To explain this difference, recall that the optimized blade is operating at a proportionately higher mean lift coefficient, and hence at a higher efficiency. This is illustrated through Figs. $15-17$. These figures present gray scale contours of the reference rotor (Fig. 15), the optimized rotor for Task 1 (Fig. 16) and the optimized rotor for Task 2 (Fig. 17), all for an advance ratio of $\mu=0.35$. In these figures bright shades represent higher lift coefficients. Figure 16 shows that the optimized rotor for Task 1 has a lift coefficient distribution similar to the reference rotor (except for a mild increase in $c_{1}$ on the retreating side). This is to be expected since both rotors are operating at the same $\mathrm{C}_{\mathrm{T}} / \sigma$ (i.e., same mean $C_{L}$ ). Figure 17 shows that the optimized rotor for Task 2 requires much higher local values of $c_{1}$ and hence is operating at a more efficient condition. This advantage will remain until the thrust requirements become high enough to drive the smaller rotor into stall. For the low speed conditions where thrust requirements are low, the optimized rotor has the advantage. The optimized rotor continues to operate at an advantage until the loads associated with high speed begin to drive it into stall.

Figure 18 presents the rotor L / D for the two rotors. These curves simply reflect the results presented in the power comparisons. The comparisons for Task 1 and Task 2 are presented. In both instances, the optimized rotor has a higher L/D than the reference rotor. In particular, the comparison for Task 2 shows a remarkable increase in efficiency. This can be directly related to the higher mean lift coefficient of the optimized blade and the corresponding increase in efficiency. The advantage drops sharply at high advance ratio where the load increases.

Figure 19 presents the predicted thrust envelope for each rotor. Both rotors are shown to be able to achieve similar levels of $C_{T} / \sigma$, since $C_{T} / \sigma$ is a measure of maximum $C_{L}$ and both rotors have the same airfoil section. However, the reference rotor has considerably more lift capacity due to its larger size. Figure 20 presents the same information converted to a load factor 
based on the thrust produced by the reference rotor operating at a $C_{T} / \sigma$ of .06 . The optimized rotor is shown here to have about half of the ultimate lifting capacity of the reference rotor.

Figures 21 and 22 present the control displacements required to achieve the rotor trim for the two tasks. These comparisons are qualitative in nature since there are no actual control margins with which to compare. Nevertheless, they provide a measure of the effects of the optimization process. Figure 21 presents the collective pitch required versus speed for the reference rotor and the optimized rotor for Task 1 and Task 2 . The figure presents the gross trends one would expect for the optimized blade alone. That is, the collective pitch required for Task 1 is less than that for Task 2. However, the comparison with the reference blade is of some interest. By simple analogy with an untwisted wing, one would expect the optimized blade to have the same collective pitch as the reference blade when operating at Task 1 and twice the collective pitch when operating at Task 2 . Figure 21 , however, shows about $25 \%$ decrease in collective for Task 1 and only about 50\% increase for Task 2 . This may be due to the redistribution of forces on the blade caused by the optimization process. However, this point needs further investigation. Figure 22 presents the longitudinal cyclic pitch required for the two rotors. This effect can be explained by reference to Figs. $15-17$. From these plots of local lift it can be seen that one feature of the optimized rotor is that it consistently shifts the load to the retreating side of the disk as compared to the reference rotor. This would necessitate an increased longitudinal cyclic depending on the magnitude of the shift.

To summarize the performance results, the optimized rotor has been shown to display an increase in overall efficiency which is achieved at the expense of ultimate lifting capacity and/or maneuvering margin.

\section{Conclusions}

The dynamic and aerodynamic performance of a rotor blade optimized to reduce only 4/rev vertical shear and blade weight was studied in detail. It was of interest to determine how well a blade designed for one flight condition and a limited number of constraints would perform at other conditions. It was also of interest to assess the performance of such a blade with respect to criteria not included while designing the blade. The program CAMRAD was used for both dynamic and aerodynamic analyses using the same modeling assumptions used to obtain the optimized blade. The behavior of the optimized rotor was compared with the reference rotor which was used as a baseline in the optimization study. The comparisons were made over a wide range of operating conditions, including speed and thrust variations which depart significantly from the optimum design condition. The dynamic behavior was assessed by comparing the blade root vibratory shears and moments that are transmitted to the rotor hub. Even though aerodynamic performance was not a design objective in the optimized blade design, an extensive aerodynamic study was undertaken for the present paper. The aerodynamic performance assessments were made based on the power required by the rotors for a given rotor task, the rotor lifting efficiencies, maximum rotor thrust envelopes and the control margins. Loads and performance were studied for the reference and the optimized rotors for two rotor tasks. Task 1 required both rotors to maintain the same $C_{T} / \sigma$ (which corresponds to the design condition) and 
Task 2 required both rotors to maintain the same $T$ (the same loading). The study yielded the following conclusions (within the context of the modeling assumptions made):

1) The optimized blade shows good dynamic and aerodynamic performance behavior at conditions other than those for which it was designed.

2) The $4 / \mathrm{rev}$ vertical shear of the optimized rotor, minimized in the optimized blade at a specific forward flight condition $(\mu=0.30)$, is lower than that of the reference blade over the entire speed range studied for Task 1 and exceeds the reference blade value for Task 2 only at higher speed.

3) Other critical vibratory blade root forces and moments, e.g., the inplane and radial shears, the flapping, lagging and torsional moments, not considered in the optimum design, are also lower for the optimized blade, than the reference blade, for the entire speed range in Task 1 and only exceeds the reference values for Task 2 at high speeds. There are occasional unexpected reductions in the flapping and lagging moments of the optimized rotor with speed for both rotor tasks.

4) The optimized blade requires lower collective pitch for both rotor tasks. Blade longitudinal flapping is higher for the optimized blade due to a shift in blade lift from the advancing to the retreating side of the disk.

5) Rotor performance (particularly efficiency) is increased even though performance was not a design criteria for the optimized blade. This was accomplished at the expense of a reduced maximum lifting capacity.

6) The total power required is lower for the optimized blade for both rotor tasks.

\section{$\underline{\text { References }}$}

1. Adelman, Howard M. and Mantay, Wayne R., "An Initiative in Multidisciplinary Optimization of Rotorcraft," NASA TM-101523, AVSCOM TM 88-B-016, October, 1988.

2. Chattopadhyay, Aditi, Walsh, Joanne L. and Riley, Michael F., "Integrated Aerodynamic/Dynamic Optimization of Helicopter Blades," Proc.

AIAA/ASME/ASCE/AHS 30th Structures, Structural Dynamics and Materials Conference, Mobile, Alabama, April 3-5, 1989. AIAA Paper No. 89-1269. Also available as NASA TM-101553, February, 1989.

3. Johnson, W., "A Comprehensive Analytical Model of Rotorcraft Aerodynamics and Dynamics," Part II: User's Manual, NASA TM-81183, June 1980.

4. Vanderplaats, G. N., "CONMIN - A FORTRAN Program for Constrained Function Minimization," User's Manual, NASA TM X-62282, August 1973.

5. Yeager, W., Mantay, W., Wilbur, M., Cramer, R., and Singleton, J., "Wind Tunnel Evaluation of an Advanced Main Rotor Blade Design for A Utility Class Helicopter, NASA TM-89129, September 1987.

6. Johnson, W., "A Comprehensive Analytical Model of Rotorcraft Aerodynamics and Dynamics," Part 1: Analysis Development, NASA TM-81182, June 1980. 
Table 1 Preassigned blade parameters

\begin{tabular}{ll}
\hline \hline Blade radius, $R(\mathrm{ft})$ & 4.685 \\
Number of blades & 4 \\
Rotational speed, $\Omega(\mathrm{rad} / \mathrm{sec})$ & 639.5 \\
Flap hinge offset/radius & 0.0534 \\
Inplane hinge offset/radius & 0.0534 \\
Maximum twist (degree) & -16 \\
Design advance ratio, $\mu$ & 0.3 \\
\hline
\end{tabular}

Table 2 Optimization Results (Ref. 2)

\begin{tabular}{|c|c|c|}
\hline & Reference blade & Optimized blade \\
\hline $\mathrm{EI}_{\mathrm{xx}}\left(\mathrm{lb}-\mathrm{ft}^{2}\right)$ & 10277.0 & 11818.0 \\
\hline $\mathrm{EI}_{\mathrm{zz}}\left(\mathrm{lb}-\mathrm{ft}^{2}\right)$ & 354.0 & 402.15 \\
\hline $\mathrm{GJ}_{r}\left(\mathrm{lb}-\mathrm{ft}^{2}\right)$ & 261.0 & 261.0 \\
\hline$k_{r}(f t)$ & 0.27 & 0.18 \\
\hline$\lambda$ & 1.0 & 1.33 \\
\hline$c_{T}(f t)$ & 0.45 & 0.28 \\
\hline $\boldsymbol{\sigma}$ & 0.122 & 0.062 \\
\hline Blade weight (lb) & 3.41 & 3.05 \\
\hline $\begin{array}{l}\text { 4/rev vertical } \\
\text { shear (lb) }\end{array}$ & 0.16 & 0.04 \\
\hline$f_{3}$ (per rev) & 3.07 & 3.28 \\
\hline $\mathrm{f}_{4}$ (per rev) & 6.76 & 6.82 \\
\hline$f_{5}$ (per rev) & 9.28 & 9.49 \\
\hline$f_{6}$ (per rev) & 12.63 & 12.51 \\
\hline $\mathrm{AI}\left(\mathrm{lb}-\mathrm{ft}^{2}\right)$ & 19.75 & 19.95 \\
\hline Thrust, T (lb) & 298.7 & 151.8 \\
\hline
\end{tabular}

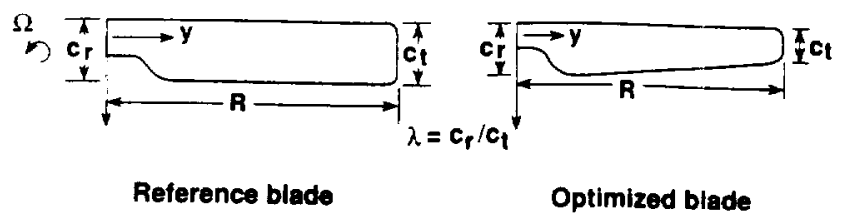

Fig. 1 Rotor blade model.

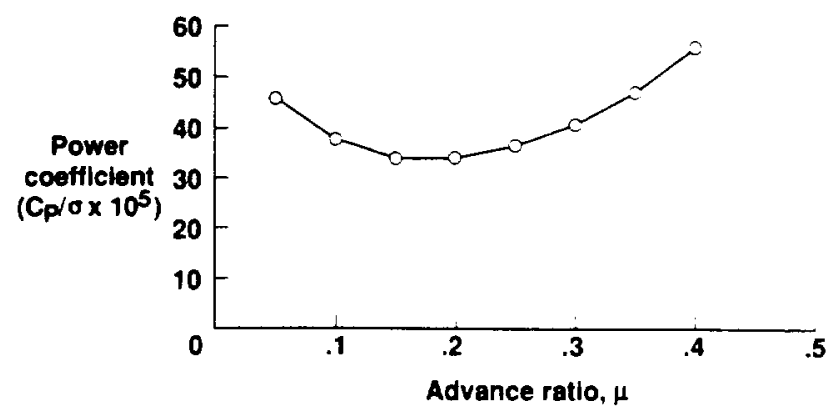

Fig. 2 Rotor power required.

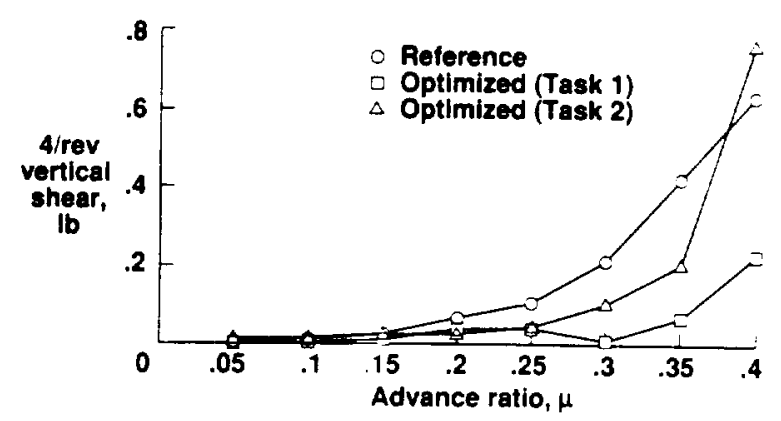

Fig. $34 / \mathrm{rev}$ vertical shear.

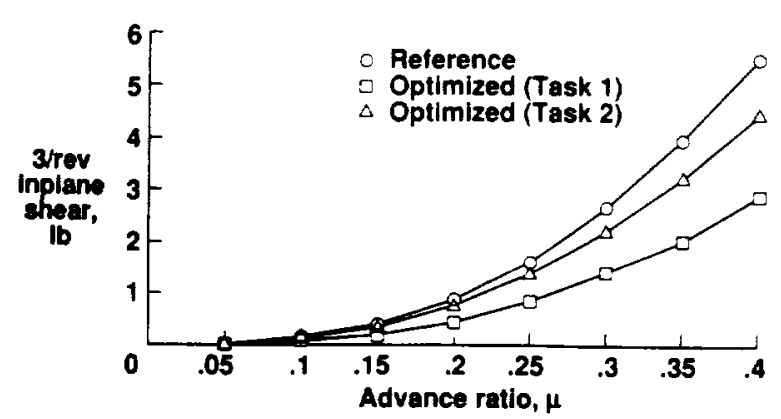

Fig. 4 3/rev inplane shear.

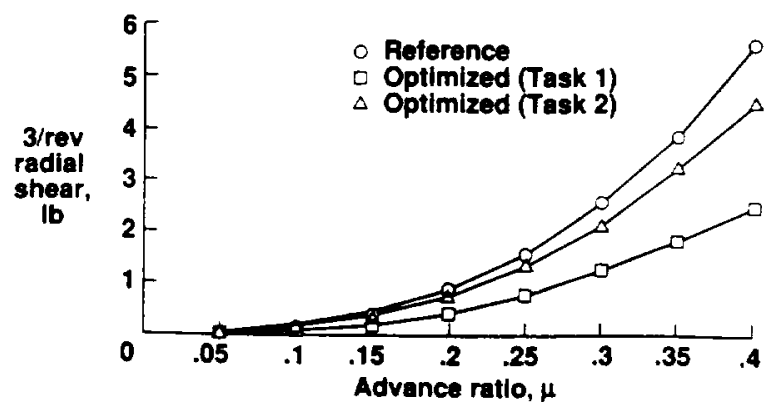

Fig. 5 3/rev radial shear. 


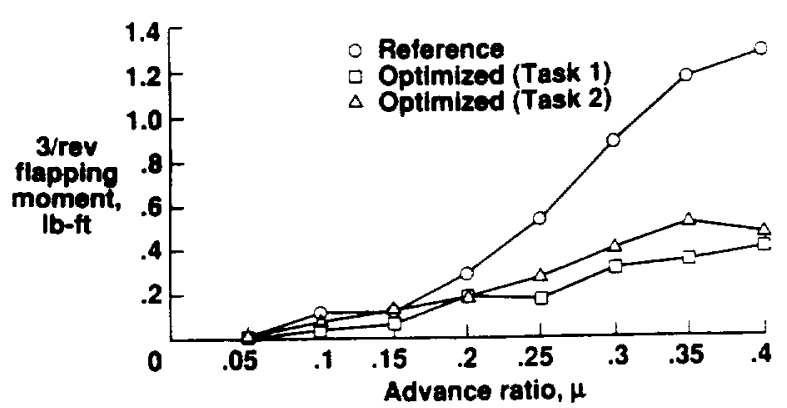

Fig. $63 /$ rev flapping moment.

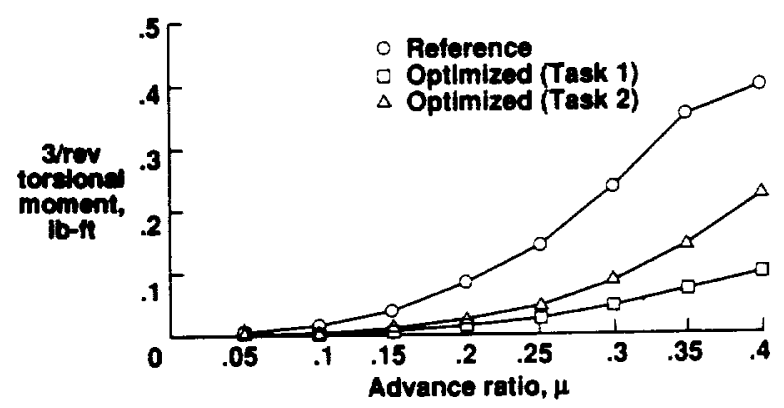

Fig. 7 3/rev torsional moment.

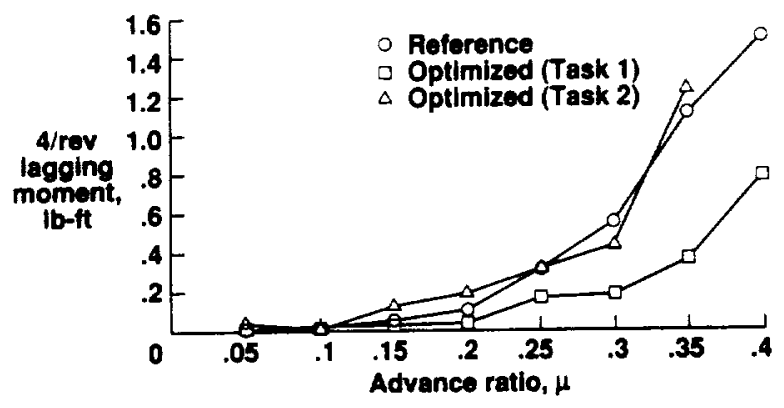

Fig. 8 4/rev lagging moment.

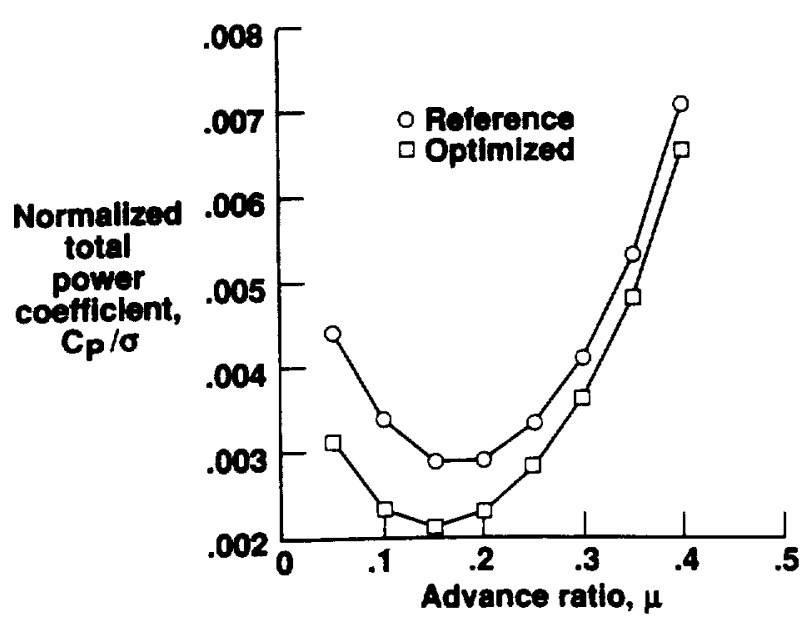

Fig. 9 Normalized total power coefficient (Task 1).

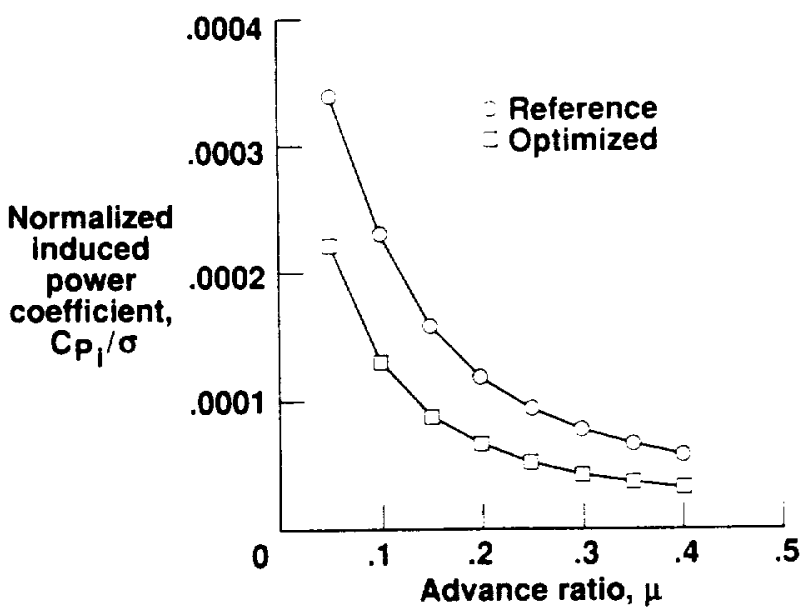

Fig. 10 Normalized induced power coefficient (Task L).

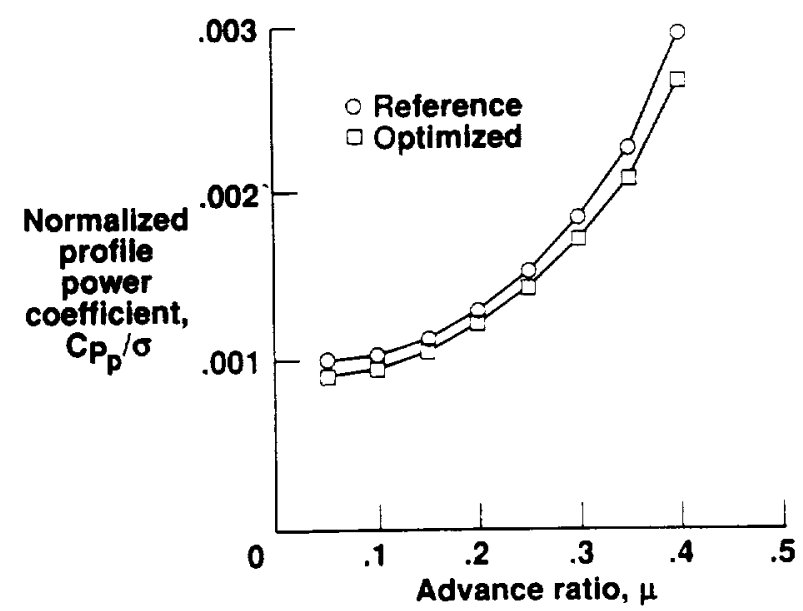

Fig. 11 Normalized profile power coefficient (Task 1).

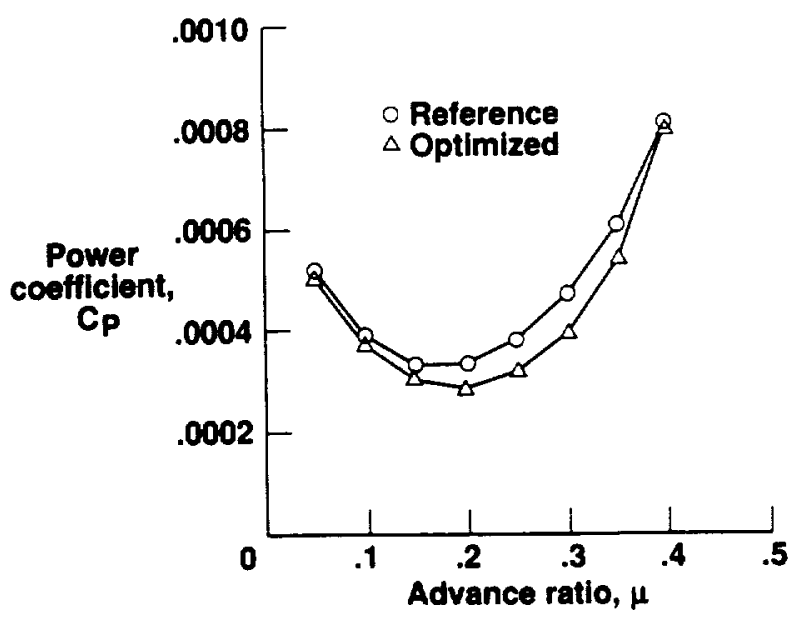

Fig. 12 Total power coefficient (Task 2). 


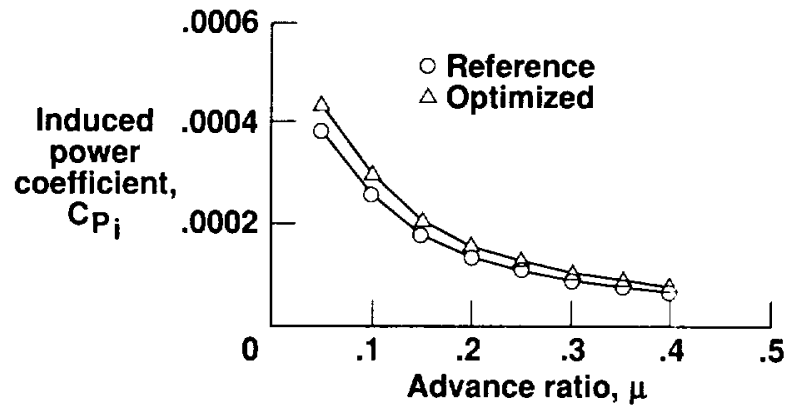

Fig. 13 Induced power coefficient (Task 2).

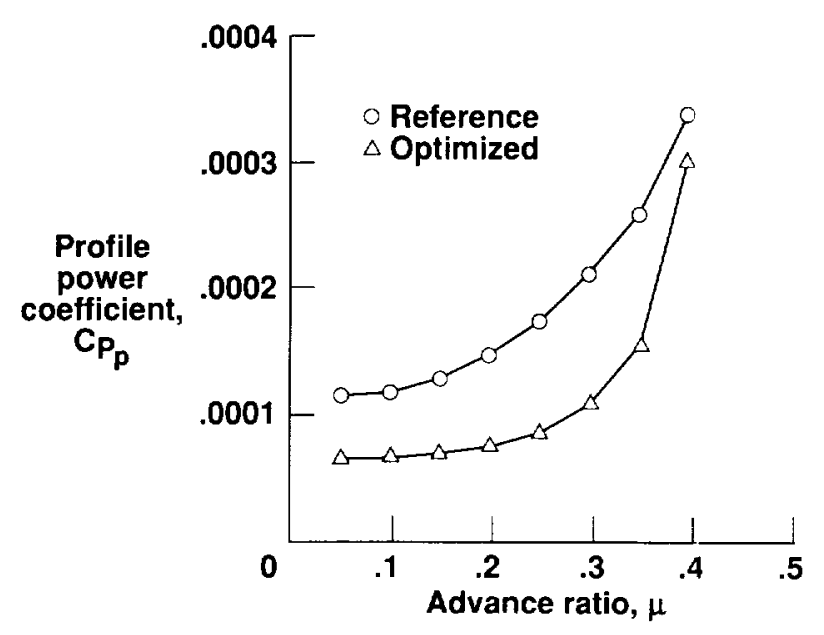

Fig. 14 Profile power coefficient (Task 2).

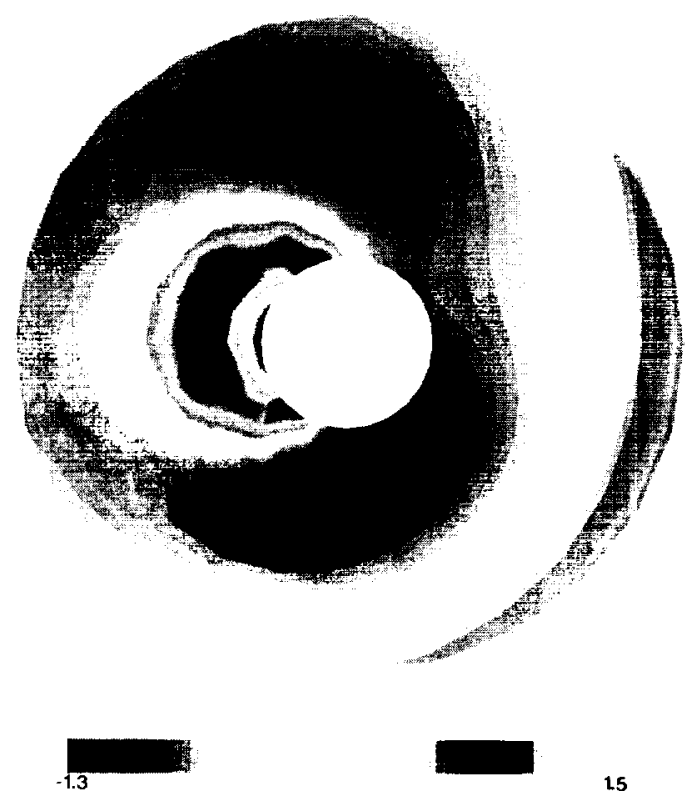

Fig. 15 Reference blade $c_{1}$ distribution.

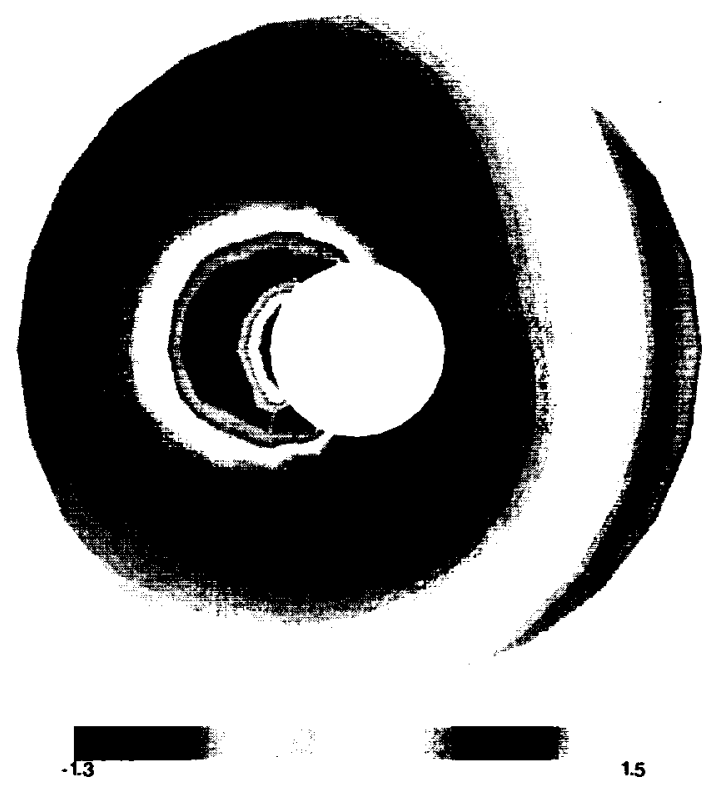

Fig. 16 Optimized blade $c_{1}$ distribution (Task 1).

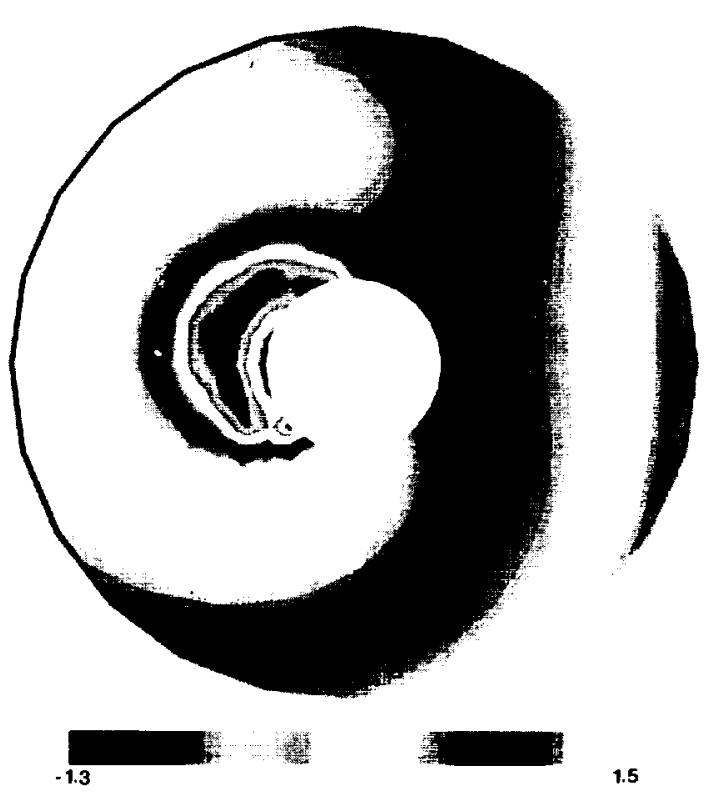

Fig. 17 Optimized blade $c_{1}$ distribution (Task 2). 


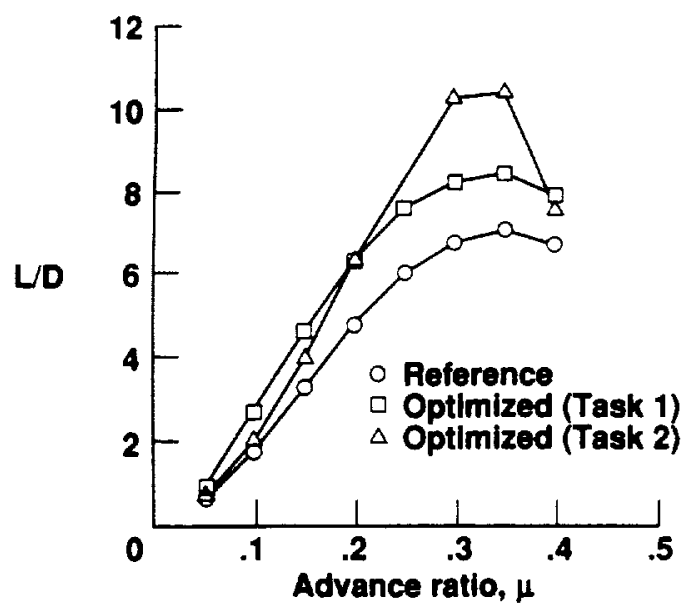

Fig. 18 Rotor L/D.

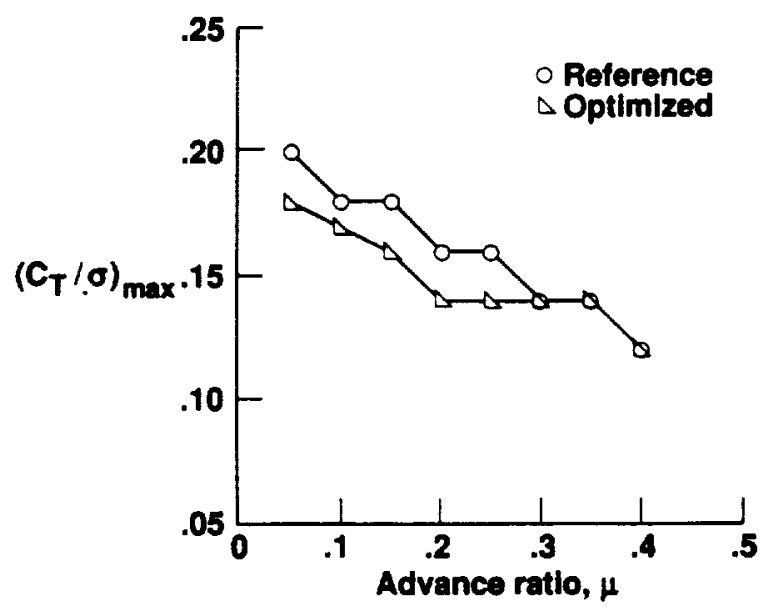

Fig. 19 Thrust limited envelope.

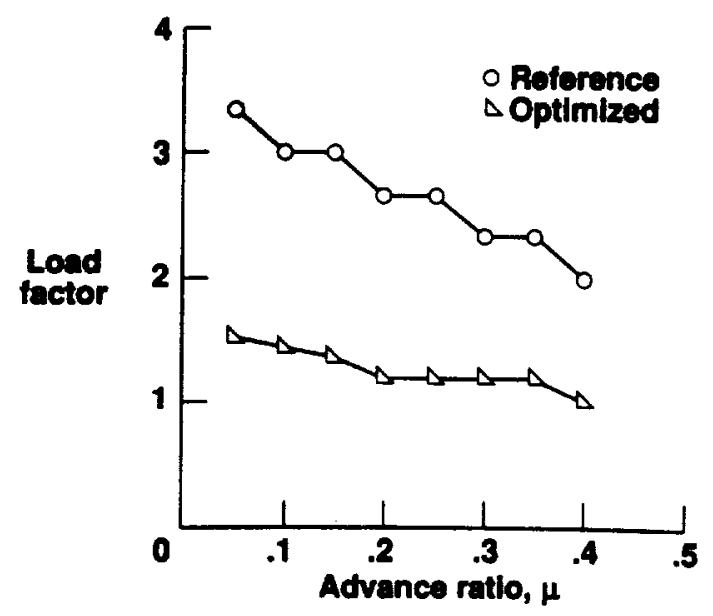

Fig. 20 Comparison of losd factor capability.

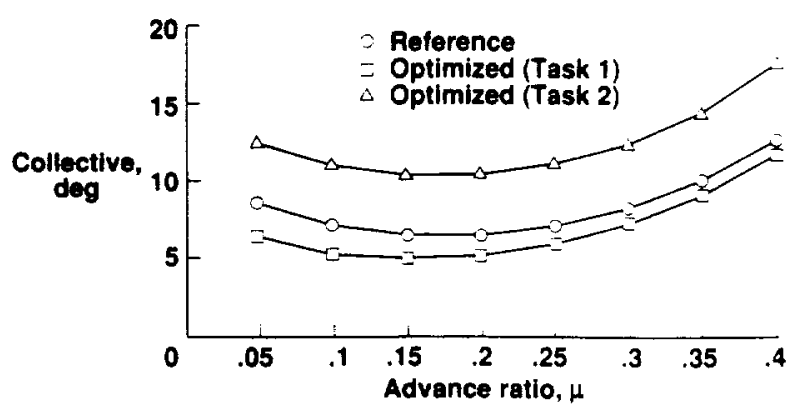

Fig. 21 Collective pitch.

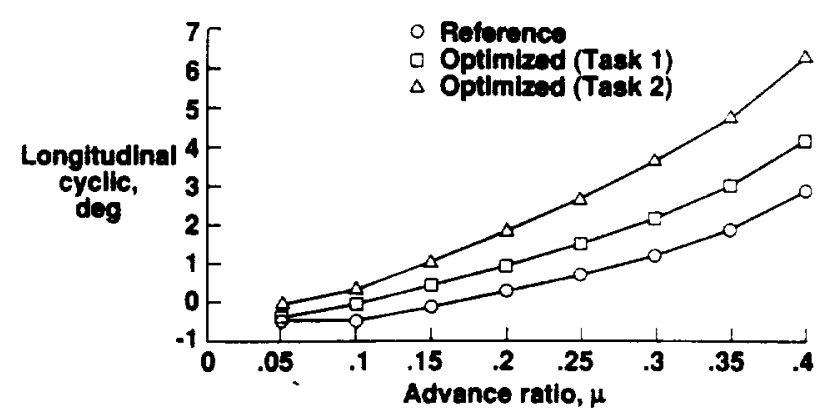

Fig. 22 Longitudinal cyclic pitch. 


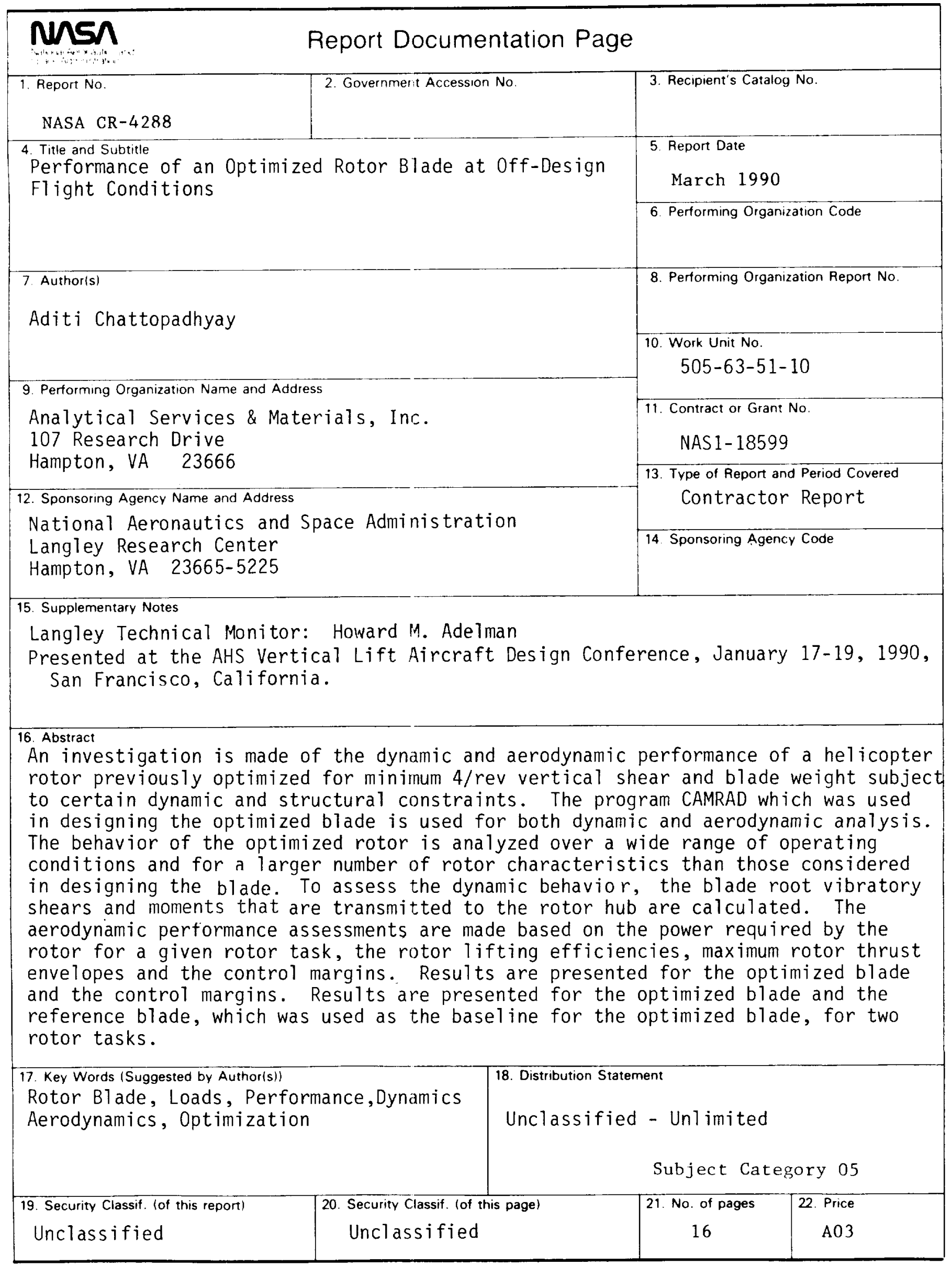


- 